\title{
Tutorial on Dissolution Calibration: An Industrial Perspective
}

G.P.Martin, D.G.Reed, L.E.Magiso M.F.Griffith and D.Ip

Merck \& Co. Inc., West Point, PA egular calibration of a dissolution apparatus in an industrial environment is appropriate for numerous reasons. These reasons include demonstrating that the apparatus is functioning properly, complying with compendial requirements and allowing the comparison of data generated on multiple formulation batches or in multiple laboratories.

Calibration normally encompasses a thorough check of mechanical properties of the apparatus, performance of the USP Apparatus Suitability Test and interpretation of the test results. Careful attention to detail at each step is critical.

Calibration requirements for USP Apparatus 1 (baskets) and 2 (paddles) are clearly stated in USP < 711> Dissolution.(1) Temperature of the medium in the pots must be maintained at $37+$ - 0.5 degrees $C$ (assure that the thermometer is calibrated). There should be no significant vibration. The vessels must conform to the listed dimensional specifications. Since few labs have the capability to measure this effectively, it makes sense to obtain vessels from a reputable vendor who can assure this. The shaft should be centered within 2 $\mathrm{mm}$. Most dissolution apparatus manufacturers have devices to assist in demonstrating this.

The absence of significant wobble can be demonstrated with a runout gauge. Alternatively, roll the shaft across a flat surface, such as a laboratory bench - there should be no gaps between the shaft and bench. Rotational speed must be within $4 \%$ of target; this can be demonstrated with a calibrated tachometer or stopwatch (after affixing a "flag" of tape to indicate when a revolution has been completed). Baskets and paddles must conform to the USP specifications. Baskets should not be misshapen; the edges of the wire mesh should be smooth and the proper gauge of mesh should be used. Paddles should be free of nicks or scars and the inert coating (if present) should be intact and not flaking. The distance between the inside bottom of the flask and the bottom of the basket or paddle should be maintained at $25+/-2 \mathrm{~mm}$.

\section{Performance of the Apparatus Suitability Test Apparatus 1 and 2}

The Apparatus Suitability Test is performed using Disintegrating (prednisone) and Nondisintegrating (salicylic acid) tablets as specified in USP $<711>$ and in the instructions which accompany the tablets obtained from the USP. The apparatus must be tested at both 50 and $100 \mathrm{rpm}$ with both types of tablets. If an apparatus is used solely with baskets or solely with paddles, it is only necessary to test for that apparatus.
For each type of calibrator tablet the instructions indicate that tablets should not be exposed to excessive humidity. In some laboratories, only freshly opened bottles of calibrator tablets are used to avoid the risk of failure due to tablets which may have been exposed to excessive humidity. A more practical alternative is to store bottles which have been opened in an area with relatively low humidity. The instructions also specify the use of deareated dissolution medium at 37 degrees $\mathrm{C}$ and a sampling time of 30 minutes. Note that the USP specification of $+/-2 \%$ for sampling time corresponds to +/36 seconds in this case.

Various techniques have been used for deaeration of dissolution medium. The USP states, "One method of deaeration is as follows: Heat the medium, while stirring gently, to about 45 degrees $\mathrm{C}$, immediately filter under vacuum using a filter having a porosity of 0.45 microns or less, with vigorous stirring, and continue stirring under vacuum for about $5 \mathrm{~min}$ utes. Other validated deaeration techniques for removal of dissolved gases may be used." Alternative methods which have been used with success include sparging with helium gas for 15 to 60 minutes or using a commercial device which pulls a vacuum across a gas-permeable membrane (such as the Erweka Dissofill).

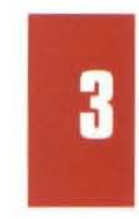




\section{Tutorial on Dissolution Calihbration ... Eont.}

Methods used for filtering samples must be checked for adsorptive loss of drug and for interference. Typically, this is accomplished by filtering aliquots of a standard solution and a blank solution of the dissolution medium, respectively. In the case of membrane filters, do not use the first $2 \mathrm{ml}$ of solution unless separate interference and recovery experiments have been carried out and demonstrate there is no significant effect from using these filters. It is advisable to subject the standard solutions to the same treatment as the samples.

For the USP Dissolution Calibrator, Disintegrating Type (Prednisone), the medium is water. A portion of the prednisone standard sufficient for the assay should be dried according to the USP instructions which accompany the reference standard. Alcohol (ethanol) (not to exceed 5\% of the total volume) may be used to bring the prednisone standard into solution. The amount of prednisone in solution is measured, using a suitably calibrated spectrophotometer, at the wavelength of maximum absorbance around $242 \mathrm{~nm}$ by comparison to a standard solution of known concentration.

For the USP Dissolution Calibrator, Nondisintegrating Type (Salicylic Acid), the medium is $0.05 \mathrm{M}$ phosphate buffer $\mathrm{pH} 7.40+/-0.05$. The buffer should be prepared as described in the USP under Buffers and Reagents, and the $\mathrm{pH}$ should be measured at room temperature.
A portion of the reference standard is dried according to USP instructions and brought into solution using an amount of alcohol not to exceed $1 \%$ of the total volume. The amount of salicylic acid in solution is measured, using a suitably calibrated spectrophotometer, at the wavelength of maximum absorbance around $296 \mathrm{~nm}$ by comparison to a standard solution of known concentration.

According to the certificate supplied with the USP Dissolution Calibrator Tablets, "The apparatus is suitable if each of the individual calculated values for each apparatus at all indicated speeds are within the specified ranges, as shown in the Table" accompanying the calibrator tablets.

The ranges included in the tables are determined by roundrobin testing in a variety of laboratories encompassing industry, regulatory agencies and the USP.(2) It should be noted that failures are not rare: a review of 2981 runs showed a $17.9 \%$ overall apparatus failure rate for baskets at $50 \mathrm{rpm}$ when tested with prednisone tablets, and $10 \%$ for paddles.(3) Indeed, there was spirited discussion on this topic at a recent USP Open Conference which included a session on dissolution testing. One topic brought up included the possibility of a Stage 2 test for Apparatus Suitability similar to that used for dissolution testing of formulations. Additionally, recommendations to reduce testing or replace current calibrator tablets with different formulations have been made.

\section{Apparatus 3}

A similar process is used to demonstrate the suitability of Apparatus 3 (Reciprocating Cylinders). Temperature must be maintained at $37+/-0.5$ degrees $\mathrm{C}$ and there must be no significant vibration. Specifications are provided for vessel geometry, and the reciprocation (dip) rate must be maintained within $5 \%$ of target. USP Drug Release Calibrator, Single Unit (Chlorpheniramine Extendedrelease Tablets) and USP Drug Release Calibrator Beads, Multiple Unit (Theophylline Extended-release Beads) are tested in each position according to the operating conditions specified by the USP.

\section{Documentation}

Laboratories should have Standard Operating Procedures in place, covering both general aspects of data generation and specifically relating to the dissolution apparatus. These should include the frequency of preventative maintenance and recalibration, and the steps to be taken in the event of a calibration failure. All pertinent information should be recorded, including lot numbers for reference standards and calibrator tablets, identification of apparatus being calibrated, other instruments used during the calibration (such as balances or spectrophotometers) and, of course, all raw data generated. This should include every calibration attempt, not just the successful ones. All calibration failures should be investigated, problems corrected and fully 
Table 1. In-house Apparatus Suitability Requirements

\begin{tabular}{|l|l|l|}
\hline Stage 1 12 & $\begin{array}{l}\text { The mean at } 30 \text { minutes is between } \\
36 \text { and } 40 \% \text { and the RSD is not more } \\
\text { than } 11 \% \text { The mean at } 60 \text { minutes is } \\
\text { between } 63 \text { and } 67 \% \text { and the RSD is } \\
\text { not more than } 9 \% .\end{array}$ \\
\hline Stage 2 & 12 & $\begin{array}{l}\text { The mean (of } 24) \text { at } 30 \text { minutes is } \\
\text { between } 34 \text { and } 42 \% \text { and the RSD is } \\
\text { not more than } 12.5 \% \text {. The mean at } \\
60 \text { minutes is between } 61 \text { and } 69 \% \\
\text { and the RSD is not more than } 10.5 \% .\end{array}$ \\
\hline
\end{tabular}

Figure 1. The Effect of Medium pH on Drug Release Profile of a Water Soluble Compound

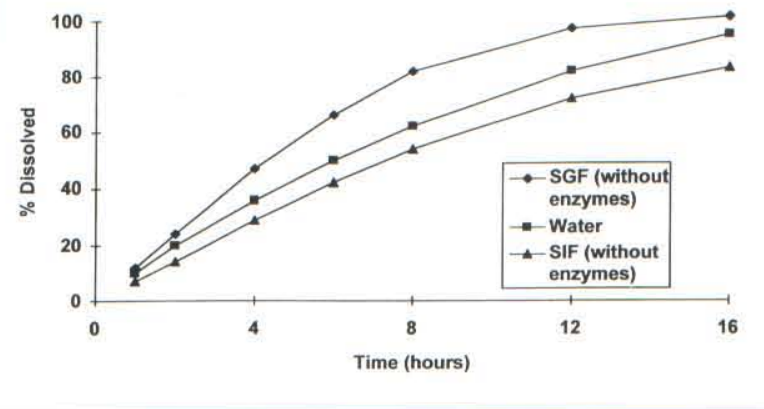

documented. As noted previously, it is not always possible to find an assignable cause for a dissolution calibration failure.

\section{Industrial Observations}

In an industrial setting, various factors can have an effect on dissolution results. Cited below are some situations which have occurred in Merck Pharmaceutical Research Laboratories which may provide some useful insight for applications in other laboratories.

\section{In-House Balibrator}

For one controlled release product, we observed that the acceptability of dissolution results varied from lab to lab, even for the same lot. Obviously, this caused concern: how do you know if a lot is acceptable, if different labs are showing different conclusions? An intensive investigation was conducted. All devices used for this testing passed the USP Apparatus Suitability Tests. However, a control lot used during the testing showed discriminating results. Round-robin testing was conducted on multiple devices at multiple sites, and statistical evaluation of the data was used to establish acceptance criteria for the dissolution apparatus for this product. The criteria, shown in Table 1, have been successful in identifying dissolution apparatuses which will provide data which can be reproduced in other laboratories.

\section{Effert of Medium pH}

It can be important to understand the $\mathrm{pH}$ dependence of the dissolution process for a given formulation. A knowledge of the $\mathrm{pH} /$ solubility profile for a compound can provide valuable information relative to sink conditions and the potential for discrimination. As part of the method development process in our laboratory, we normally investigate medium of various $\mathrm{pH}$ 's, typically including simulated gastric fluid (without enzymes) and simulated intestinal fluid (without enzymes). Dissolution profile curves for a sustained release product containing a water soluble drug are shown in Figure 1.

\section{Effert of Sinker Type}

For some dosage forms, including capsules, it is necessary to use a sinker to prevent floating, which can lead to irreproducible results. The USP specifies a wire coil or other suitable device. For one capsule formulation, our laboratory evaluated wire coils, a commercial device (multipronged plastic) and paper clips. Results, shown in Figure 2, (page 6) indicated that there was no significant difference between the wire coil and the commercial device, but that the paper clip produced faster dissolution. The method was written to specify that either wire coils or the commercial device could be used.

\section{Deaeration}

If the dissolution medium is not deaerated, small bubbles may form on surfaces during the dissolution process, including the dosage form, the vessel and the paddle or basket. This can lead to variability in results and must be evaluated empirically for each dosage form. To determine the effect on one sustained release formulation, we compared results generated using different types of deaeration to results when the medium was not deaerated. The profiles, shown in Figure 3, indicated that there was no significant effect from moderate deaeration, such as that described in the USP or helium sparging for 45 minutes. However, when the medium was sparged with helium for 12 


\section{Tutorial on Dissolution Ealibration ... Eont.}
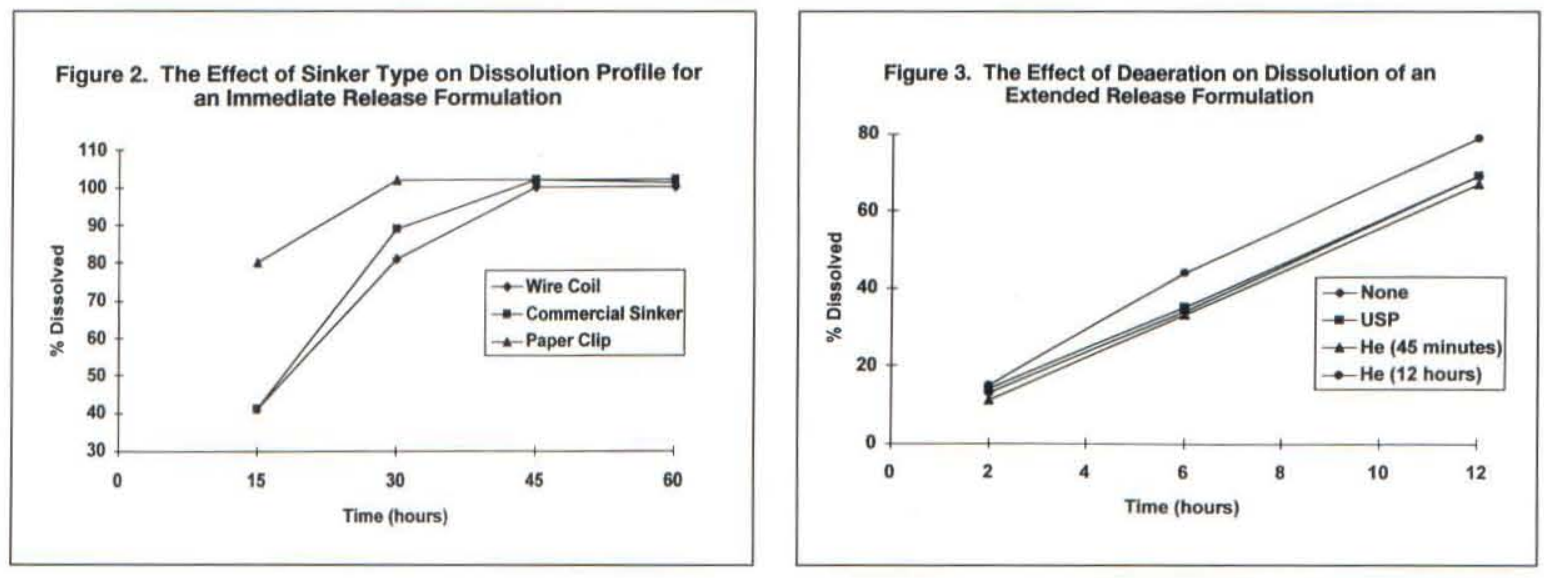

hours (overnight), the drug dissolved faster. Hence, acceptable means of deaeration (none, USP or 45 minute helium sparging ) were specified in the method.

\section{Eonclusion}

As can be seen from this discussion, dissolution testing is more complex than one might initially expect. Recently, there has been discussion on the possibility of using alternative formulations for calibration which may be more discriminating than the current ones $(4,5)$ as well as reducing the amount of testing necessary for calibration of an apparatus (6). Having properly calibrated baths is one way to assure that consistent data will be generated, but it is necessary to have a good understanding of the product being tested in order to be able to interpret similarities and differences among dissolution data for different lots of a given product, especially if they are generated in different laboratories.

\section{References}

1. The United States Pharmacopoeia, 23rd revision, Second Supplement, 2707-2711 (1995).

2. Pharmaceutical Research and Manufacturers of America, Pharmacopeial Forum, 20(5), 8177-8201 (1994).

3.A.S.Achanta, V.A.Gray, T.L.Cecil and L.T.Grady, Drug Development and Industrial Pharmacy, 21(10), 1171-1182 (1995).

\section{J.F.Hamilton,}

T.W. Moore and C.M.Kerner, Pharmacopeial Forum, 21(5), 1383-1386 (1995).

5.T.W.Moore,
J.F.Hamilton and C.M.Kerner, Pharmacopeial Forum, 21(5), 13871396 (1995).

6. R.F.Lindaurer, Pharmacopeial Forum, 21(5), 1397-1402 (1995).

\section{B}

